\title{
Sufficient Conditions for the Convergence of a Class of Nonlinear Distributed Consensus Algorithms *
}

\author{
Amir Ajorlou ${ }^{\text {a }}$, Ahmadreza Momeni ${ }^{\text {a }}$, Amir G. Aghdam ${ }^{\text {a }}$ \\ ${ }^{a}$ Department of Electrical and Computer Engineering, Concordia University, Montréal, Québec, Canada
}

\begin{abstract}
This paper is concerned with the convergence of a class of continuous-time nonlinear consensus algorithms for single integrator agents. In the consensus algorithms studied here, the control input of each agent is assumed to be a state-dependent combination of the relative positions of its neighbors in the information flow graph. Using a novel approach based on the smallest order of the nonzero derivative, it is shown that under some mild conditions the convex hull of the agents has a contracting property. A set-valued LaSalle-like approach is subsequently employed to show the convergence of the agents to a common point. The results are shown to be more general than the ones reported in the literature in some cases. An illustrative example demonstrates how the proposed convergence conditions can be verified.
\end{abstract}

\section{Introduction}

Consensus is one of the most important specifications in multi-agent control applications. Early work on the consensus problem can be traced back to the field of computer science and distributed computations [12]. In the classical consensus problem, it is desired to find a state update rule for the agents such that some quantity of interest in every agent converges to a common value in the steady state. Further results on this subject are presented in the literature in the past few years; e.g., see $[13,14]$. In [13], linear timeinvariant (LTI) consensus protocols are proposed for multiagent systems subject to switching communication topologies and time-delay. The work [14] proposes both discrete and continuous time consensus protocols for a group of agents which exchange information over limited and unreliable communication links with time-varying topology. Recently, some algorithms have been proposed in the literature which guarantee the connectivity of the underlying network of agents $[4,2,1]$. Collision avoidance is another important problem concerning the consensus algorithms, and has been addressed in a number of papers $[11,15,2]$.

In all of the above-mentioned algorithms, the stability of the system under some control strategy is to be determined, typically by finding an appropriate Lyapunov function $[11,15,4,2]$. However, constructing a proper Lyapunov function is known to be cumbersome, in general. Motivated by this shortcoming, some recent papers consider the stability of general distributed consensus algorithms [9,10,8,7]. Graphical conditions are presented in [9] for the exponential

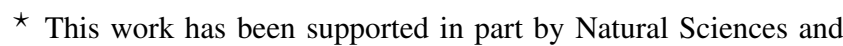
Engineering Research Council of Canada (NSERC) under Grant STPGP-364892-08, and in part by Motion Metrics Int'l Corp.
}

stability of a class of continuous linear time-varying (LTV) systems whose state-space matrix is Metzler with zero row sums. In [10], the convergence of discrete-time nonlinear consensus algorithms with time-dependent communication links is shown under a convexity assumption and some conditions on the communication graph. As the continuoustime counterpart of [10], [8] studies the state agreement for coupled nonlinear differential equations with switching vector fields and topology. It is shown that under a strict sub-tangentiality condition and uniformly quasi-strongly connectedness of the interaction digraph, the system has the property of asymptotic state agreement. Somewhat relaxed conditions for the case of a static interaction digraph are presented in [7]. Nonlinear consensus algorithms arise in applications where other design criteria such as connectivity preservation and collision avoidance are to be satisfied during the convergence to a consensus $[4,2,1]$.

The present paper studies the convergence of a class of continuous-time nonlinear consensus algorithms for single integrator agents. The information flow graph of the agents is assumed to be static and directed. The control input of each agent is considered as a state-dependent combination of the relative positions of its neighbors in the information flow graph. Sufficient conditions are provided which guarantee the convergence of the agents to a common point for this class of consensus algorithms. It is shown that under some mild conditions, the convex hull of the agents has a contracting property. This property is used later to prove the convergence of the agents to a common point. The proposed convergence conditions are more general than the ones reported in $[7,8]$ under the additional assumption that the weights are analytic for a static interaction graph. The verification of the proposed convergence conditions is illustrated via a leader-based consensus example. 


\section{Problem Formulation}

Definition 1 For a smooth function $f: \mathbb{R} \rightarrow \mathbb{R}$, the index of $f$ at time $t$, denoted by $\rho(f(t))$, is defined as the smallest natural number $n$ for which $f^{(n)}(t) \neq 0$. Similarly, the extended index of $f$ at time $t$, denoted by $\tilde{\rho}(f(t))$, is defined as the smallest nonnegative integer $n$ for which $f^{(n)}(t) \neq 0$, where $f^{(0)}(t)$ is defined to be $f(t)$.

Definition 2 A set-valued function $S(\cdot)$ is said to be nested if for every $t_{1}, t_{2} \in \mathbb{R}$, where $0 \leq t_{1} \leq t_{2}$, the relation $S\left(t_{2}\right) \subseteq$ $S\left(t_{1}\right)$ holds.

Definition 3 A digraph $G$ is said to be quasi-strongly connected if for every two distinct vertices $u$ and $v$ of $G$, there is a vertex from which both $u$ and $v$ are reachable (see [3]).

Definition 4 A group of agents $1, \ldots, n$ is said to converge to a consensus if $q_{i}(t) \rightarrow \bar{q}$ as $t \rightarrow \infty$ for any $i \in \mathbb{N}_{n}:=$ $\{1, \ldots, n\}$, where $q_{i}(t) \in \mathbb{R}^{m}$ denotes the state of agent $i$ at time $t$, and $\bar{q}$ is a constant.

Definition 5 A family $\mathscr{A}=\left\{A_{\alpha}\right\}_{\alpha \in I}$ of subsets of a set $X$ is said to have the finite intersection property if every finite sub-family $\left\{A_{1}, A_{2}, \ldots, A_{n}\right\}$ of $\mathscr{A}$ satisfies $\bigcap_{i=1}^{n} A_{i} \neq \emptyset$ (see [5]).

Consider a set of $n$ agents in the 2D plane with single integrator dynamics, i.e.

$$
\dot{q}_{i}(t)=u_{i}(t), \quad i \in \mathbb{N}_{n}
$$

where $q_{i}(t) \in \mathbb{R}^{2}$ represents the position of agent $i$ at time $t$, and $u_{i}$ is the corresponding control signal. Note that for brevity, the time argument is omitted hereafter in all time-dependent functions, wherever it is not necessary. Denote with $G=(V, E)$ the information flow graph, with $V=\{1, \ldots, n\}$ representing the set of $n$ vertices (associated with the $n$ agents), and $E \subseteq V \times V$ representing the corresponding edges. The information flow graph $G$ is assumed to be static and directed. There is a directed edge from vertex $j$ to vertex $i$ in $G$ if and only if $(j, i) \in E$. The set of neighbors of vertex $i$ in $G$ is defined as $N_{i}=\{j \mid(j, i) \in E\}$, and its indegree is denoted by $d_{i}=\left|N_{i}\right|$. Each agent is only allowed to incorporate its own position and the position of its neighbors in its control law. In this paper, the distributed control laws of the following form are considered

$$
u_{i}=-\sum_{j \in N_{i}} \beta_{i j} \times\left(q_{i}-q_{j}\right), \quad i \in \mathbb{N}_{n}
$$

where the coefficients $\beta_{i j}: \mathbb{R}^{2\left(d_{i}+1\right)} \rightarrow \mathbb{R}, i \in \mathbb{N}_{n}, j \in N_{i}$, are state-dependent. More specifically, each coefficient $\beta_{i j}$ is a function of the position of agent $i$ and the positions of the neighbors of agent $i$ in $G$. The main contribution of this paper is to present sufficient conditions on the coefficients $\beta_{i j}$ in (2), which guarantee the convergence of the agents to a consensus.

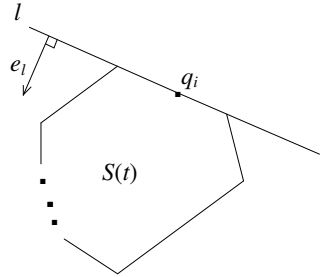

Fig. 1. $S(t)$ is the convex hull of the agents at time $t, q_{i}$ is the position of an agent on $l$, and $e_{l}$ is the unit vector perpendicular to $l$ in the direction of the half-plane containing $S(t)$.

\section{Sufficient Conditions for Convergence}

The aim of this section is to show that under the following assumptions on the coefficients $\beta_{i j}$ in (2), the agents converge to a consensus.

Assumption 1 The state-dependent coefficients $\beta_{i j}$ in (2) are analytic, real and nonnegative for any $i \in \mathbb{N}_{n}$ and $j \in N_{i}$.

Assumption 2 The system (1) with the control law of the form (2) has no solution in which the convex hull of the agents is not a singleton and is fixed, with at least one agent being fixed at each vertex.

Lemma 1 Consider a function $f: \mathbb{R} \rightarrow \mathbb{R}$ with the property that $f^{\rho(f(t))}(t)>0$, for some $t$. Then, there exists $\delta>0$ such that $f(t)<f(t+\tau), \forall \tau \in(0, \delta]$. Similarly, if $f^{\rho(f(t))}(t)<0$, then there exists $\delta>0$ for which $f(t)>f(t+\tau), \forall \tau \in(0, \delta]$.

Proof. The proof is straightforward, and is omitted here.

Denote with $S(t)$ the convex hull of the agents at time $t$, i.e. $S(t)=\operatorname{Conv}\left(\left\{q_{i}(t) \mid i \in \mathbb{N}_{n}\right\}\right)$. In what follows, a few lemmas are presented in order to prove the nestedness property for $S(t)$. To this end, it is required to investigate the behavior of the agents on the boundary of $S(t)$. Consider a line $l$ which intersects $S(t)$ at some time $t \geq 0$, but does not pass through it. Note that this intersection will be either an edge or a vertex of $S(t)$ (see Fig. 1 for the case when the intersection is an edge). Denote with $e_{l}$ the unit vector perpendicular to $l$, in the direction of the half-plane containing $S(t)$. Define $f_{l}: \mathbb{R}^{2} \rightarrow \mathbb{R}$ as $f_{l}(x)=<x, e_{l}>$, i.e., the projection of $x$ on $e_{l}$. Let agent $i$ be on $l$ at time $t$. Denote with $N_{i}^{l}(t)$ the set of those neighbors of $i$ lying on $l$, and with $\bar{N}_{i}^{l}(t)$ the set of those neighbors not lying on $l$. Now, define $\eta_{i 1}^{l}(t)$ and $\eta_{i 2}^{l}(t)$ as follows:

$$
\eta_{i 1}^{l}(t)= \begin{cases}\min _{j \in N_{i}^{l}(t)}\left\{\tilde{\rho}\left(\beta_{i j}\right)+\rho\left(f_{l}\left(q_{j}\right)\right)\right\}, & N_{i}^{l}(t) \neq \emptyset \\ \infty, & N_{i}^{l}(t)=\emptyset\end{cases}
$$

and

$$
\eta_{i 2}^{l}(t)= \begin{cases}\min _{j \in \bar{N}_{i}^{l}(t)}\left\{\tilde{\rho}\left(\beta_{i j}\right)\right\}, & \bar{N}_{i}^{l}(t) \neq \emptyset \\ \infty, & \bar{N}_{i}^{l}(t)=\emptyset\end{cases}
$$


where in calculating $\tilde{\rho}\left(\beta_{i j}\right), \beta_{i j}$ is regarded as an implicit function of time. It is straightforward to verify that $\eta_{i 1}^{l}(t) \geq 1$ and $\eta_{i 2}^{l}(t) \geq 0$. Define also $\eta_{i}^{l}(t)=\min \left\{\eta_{i 1}^{l}(t), \eta_{i 2}^{l}(t)\right\}$.

Lemma 2 Consider a line $l$ which intersects $S(t)$ at some time $t \geq 0$, but does not pass through it. Assume that $q_{i}(t) \in l$, for some $i \in \mathbb{N}_{n}$. Then, the following statements are true: i) If $\eta_{i}^{l}=0$, then $f_{l}\left(\dot{q}_{i}\right)>0$.

ii) If $\eta_{i}^{l} \geq 1$, then $f_{l}\left(q_{i}^{(k)}\right)=0$, for $k=1, \ldots, \eta_{i}^{l}$.

Proof. Part (i): First, note that $f_{l}\left(q_{j}-q_{i}\right)$ is equal to zero for any $j \in N_{i}^{l}$, and is strictly positive for any $j \in \bar{N}_{i}^{l}$. Also, $\beta_{i j} \geq 0$ for any $j \in N_{i}$, according to Assumption 1. The relation $\eta_{i}^{l}=0$ yields $\eta_{i 2}^{l}=0$, which implies that $\bar{N}_{i}^{l} \neq \emptyset$, and that there exists an agent $v \in \bar{N}_{i}^{l}$ for which $\beta_{i v}>0$. Therefore, using (1) and (2) one can write

$$
f_{l}\left(\dot{q}_{i}\right)=\sum_{j \in \bar{N}_{i}^{l}} \beta_{i j} f_{l}\left(q_{j}-q_{i}\right) \geq \beta_{i v} f_{l}\left(q_{v}-q_{i}\right)>0
$$

Part (ii): It is straightforward to show that

$$
f_{l}\left(q_{i}^{(k+1)}\right)=\sum_{j \in N_{i}} \sum_{r=0}^{k} \beta_{i j}^{(k-r)}\left(f_{l}\left(q_{j}^{(r)}\right)-f_{l}\left(q_{i}^{(r)}\right)\right)\left(\begin{array}{l}
k \\
r
\end{array}\right)
$$

where $\beta_{i j}^{(k-r)}$ is the $(k-r)^{t h}$ derivative of $\beta_{i j}$ with respect to time (note that $\beta_{i j}$ is an implicit function of time). Assume now $k<\eta_{i}^{l}$; this means that $k-r<\eta_{i}^{l} \leq \eta_{i 2}^{l}$, and hence $\beta_{i j}^{(k-r)}=0$ for $j \in \bar{N}_{i}^{l}$. On the other hand, since $k<\eta_{i}^{l} \leq$ $\eta_{i 1}^{l}$, one can easily show that $\beta_{i j}^{(k-r)} f_{l}\left(q_{j}^{(r)}\right)=0$, for $j \in N_{i}^{l}$ and $1 \leq r \leq k$. Using these results along with the fact that $f_{l}\left(q_{j}-q_{i}\right)=0$ for $j \in N_{i}^{l}$, equation (6) reduces to

$$
f_{l}\left(q_{i}^{(k+1)}\right)=-\sum_{j \in N_{i}^{l}} \sum_{r=1}^{k} \beta_{i j}^{(k-r)} f_{l}\left(q_{i}^{(r)}\right)\left(\begin{array}{c}
k \\
r
\end{array}\right)
$$

The rest of the proof follows by a simple induction.

Lemma 3 Consider a line $l$ which intersects $S(t)$ at some time $t \geq 0$, but does not pass through it. Assume that $q_{i}(t) \in l$, for some $i \in \mathbb{N}_{n}$. If $\rho\left(f_{l}\left(q_{i}\right)\right)<\infty$, then $f_{l}\left(q_{i}^{\left(\rho\left(f_{l}\left(q_{i}\right)\right)\right)}\right)>0$.

Proof. Since $\rho\left(f_{l}\left(q_{i}\right)\right)<\infty$, thus it is implied from Lemma 2 that $\eta_{i}^{l}<\infty$. To continue the proof, first some important properties of $f_{l}\left(q_{i}^{\left(\eta_{i}^{l}+1\right)}\right)$ are characterized assuming $1 \leq$ $\eta_{i}^{l}<\infty$. Using Lemma 2 and taking an approach similar to the one used to derive (7) from (6), one can show that

$$
\begin{aligned}
f_{l}\left(q_{i}^{\left(\eta_{i}^{l}+1\right)}\right) & =\sum_{j \in N_{i}^{l}} \sum_{r=1}^{\eta_{i}^{l}} \beta_{i j}^{\left(\eta_{i}^{l}-r\right)} f_{l}\left(q_{j}^{(r)}\right)\left(\begin{array}{c}
\eta_{i}^{l} \\
r
\end{array}\right) \\
& +\sum_{j \in \bar{N}_{i}^{l}} \beta_{i j}^{\left(\eta_{i}^{l}\right)} f_{l}\left(q_{j}-q_{i}\right)
\end{aligned}
$$

There are three possible cases for $\eta_{i}^{l}, \eta_{i 1}^{l}$, and $\eta_{i 2}^{l}$ : Case (i): $\eta_{i}^{l}=\eta_{i 2}^{l}<\eta_{i 1}^{l}$. In this case, (8) reduces to

$$
f_{l}\left(q_{i}^{\left(\eta_{i}^{l}+1\right)}\right)=\sum_{\substack{j \in \bar{N}_{i}^{l} \\ \tilde{\rho}\left(\beta_{i j}\right)=\eta_{i}^{l}}} \beta_{i j}^{\left(\eta_{i}^{l}\right)} f_{l}\left(q_{j}-q_{i}\right)
$$

On the other hand, the relation $\tilde{\rho}\left(\beta_{i j}\right)=\eta_{i}^{l} \geq 1$ implies that $\beta_{i j}=0$. If $\beta_{i j}^{\left(\tilde{\rho}\left(\beta_{i j}\right)\right)}<0$, then it results from Lemma 1 that $\beta_{i j}$ is negative in a right neighborhood of $t\left(\beta_{i j}\right.$ is regarded here as an implicit function of time, as noted earlier). However, this is in contradiction with Assumption 1; therefore $\beta_{i j}^{\left(\tilde{\rho}\left(\beta_{i j}\right)\right)}>0$, and it results from (9) that $f_{l}\left(q_{i}^{\left(\eta_{i}^{l}+1\right)}\right)>0$. Case (ii): $\eta_{i}^{l}=\eta_{i 1}^{l}<\eta_{i 2}^{l}$. In this case, (8) reduces to

$$
f_{l}\left(q_{i}^{\left(\eta_{i}^{l}+1\right)}\right)=\sum_{\substack{j \in N_{i}(l) \\
\tilde{\rho}\left(\beta_{i j}\right)+\rho\left(f_{l}\left(q_{j}\right)\right)=\eta_{i}^{l}}} \beta_{i j}^{\left(\tilde{\rho}\left(\beta_{i j}\right)\right)} f_{l}\left(q_{j}^{\left(\rho\left(f_{l}\left(q_{j}\right)\right)\right)}\right)\left(\begin{array}{c}
\eta_{i}^{l} \\
\tilde{\rho}\left(\beta_{i j}\right)
\end{array}\right)
$$

Similar to case (i), it can be shown that $\beta_{i j}^{\left(\tilde{\rho}\left(\beta_{i j}\right)\right)}>0$.

Case (iii): $\eta_{i}^{l}=\eta_{i 1}^{l}=\eta_{i 2}^{l}$. It results from (8) in this case that $f_{l}\left(q_{i}^{\left(\eta_{i}^{l}+1\right)}\right)$ can be written as the summation of the righthand sides of (9) and (10), and hence

$$
f_{l}\left(q_{i}^{\left(\eta_{i}^{l}+1\right)}\right)>\sum_{\substack{j \in N_{i}(l) \\
\tilde{\rho}\left(\beta_{i j}\right)+\rho\left(f_{l}\left(q_{j}\right)\right)=\eta_{i}^{l}}} \beta_{i j}^{\left(\tilde{\rho}\left(\beta_{i j}\right)\right)} f_{l}\left(q_{j}^{\left(\rho\left(f_{l}\left(q_{j}\right)\right)\right)}\right)\left(\begin{array}{c}
\eta_{i}^{l} \\
\tilde{\rho}\left(\beta_{i j}\right)
\end{array}\right)
$$

From the results presented in cases (ii) and (iii), one can easily conclude that if $\eta_{i}^{l}=\eta_{i 1}^{l}$, then

$$
f_{l}\left(q_{i}^{\left(\eta_{i}^{l}+1\right)}\right) \geq \sum_{\substack{j \in N_{i}(l) \\ \tilde{\rho}\left(\beta_{i j}\right)+\rho\left(f_{l}\left(q_{j}\right)\right)=\eta_{i}^{l}}} \alpha_{i j} f_{l}\left(q_{j}^{\left(\rho\left(f_{l}\left(q_{j}\right)\right)\right)}\right)
$$

where $\alpha_{i j}$ 's are positive coefficients. It is desired now to use induction on $\rho\left(f_{l}\left(q_{i}\right)\right)$ together with the results developed thus far to prove the lemma. For $\rho\left(f_{l}\left(q_{i}\right)\right)=1$, the proof is straightforward using Lemma 2. Assume now that the statement of the lemma holds for $\rho\left(f_{l}\left(q_{i}\right)\right) \leq k$, for some $k \geq 1$; it is desired to prove that it holds for $\rho\left(\bar{f}_{l}\left(q_{i}\right)\right)=k+1$ as well. Note first that Lemma 2 implies $1 \leq \eta_{i}^{l}<k+1$. If $\eta_{i 2}^{l}<\eta_{i 1}^{l}$, then it results from case (i) as well as Lemma 2 that $\rho\left(f_{l}\left(q_{i}\right)\right)=\eta_{i}^{l}+1$ and $f_{l}\left(q_{i}^{\left(\eta_{i}^{l}+1\right)}\right)>0$. If on the other hand $\eta_{i 2}^{l} \geq \eta_{i 1}^{l}$ (i.e. $\eta_{i}^{l}=\eta_{i 1}^{l}$ ), then (12) holds. Moreover, for any $j$ in the summation domain of (12), the relation $\rho\left(f_{l}\left(q_{j}\right)\right) \leq \eta_{i}^{l}<k+1$ holds, and hence the assumption of induction yields $f_{l}\left(q_{j}^{\left(\rho\left(f_{l}\left(q_{j}\right)\right)\right)}\right)>0$. It is concluded from this along with (12) that $f_{l}\left(q_{i}^{\left(\eta_{i}^{l}+1\right)}\right)>0$, from which it is also implied (using Lemma 2) that $\rho\left(f_{l}\left(q_{i}\right)\right)=\eta_{i}^{l}+1$. This completes the proof. 
Corollary 1 Consider a line $l$ which intersects $S(t)$ at some time $t \geq 0$, but does not pass through it. Assume that $q_{i}(t) \in l$, for some $i \in \mathbb{N}_{n}$. Then, $\rho\left(f_{l}\left(q_{i}\right)\right)=\eta_{i}^{l}+1$.

Proof. The proof follows directly from Lemma 3 (as its byproduct).

Lemma 4 Consider a line $l$ which intersects $S(t)$ at some time $t \geq 0$, but does not pass through it. Given $q_{i}(t) \in l$, if $f_{l}\left(q_{i}(t)\right)$ has a finite index, then there exists $\delta_{i}>0$ such that for any $\tau \in\left(0, \delta_{i}\right]$ the inequality $f_{l}\left(q_{i}(t)\right)<f_{l}\left(q_{i}(t+\tau)\right)$ holds; otherwise, $f_{l}\left(\dot{q}_{i}\right) \equiv 0$.

Proof. This can be easily proved using Lemma 3, and on noting that $\beta_{i j}$ 's are analytic, and hence, so are $q_{i}$ and $f_{l}\left(q_{i}\right)$.

Theorem 1 Under Assumption 1, the convex hull of the agents is nested.

Proof. Consider the agents at any arbitrary time $t \geq 0$. By applying Lemma 4 to all the edges on the boundary of $S(t)$, one can easily show that there exists $\delta(t)>0$ such that $q_{i}(t+\tau) \in S(t)$, for all $i \in \mathbb{N}_{n}$ and $\tau \in[0, \delta(t)]$, implying that $S(t+\tau) \subseteq S(t)$, for any $\tau \in[0, \delta(t)]$. The rest of the proof is straightforward and is omitted due to space limitations.

Theorem 2 Consider a set of $n$ agents in the $2 D$ plane with the dynamics of the form (1), evolved under the control laws given by (2). Under Assumptions 1-2, the agents converge to a consensus.

Proof. Since $S(t)$ is nested, the agents remain in $S(0)$ at all times. Define $\mu_{1}(q(t))$ and $\mu_{2}(q(t))$ as the area and the diameter of $S(t)$, respectively, where $q(t)=\left(q_{1}(t), \ldots, q_{n}(t)\right)$. Clearly, $\mu_{1}$ and $\mu_{2}$ are bounded and nonincreasing (note that $S(t)$ is nested) but not necessarily differentiable. Let $\lim _{t \rightarrow \infty} \mu_{1}(q(t))=a_{1}$ and $\lim _{t \rightarrow \infty} \mu_{2}(q(t))=a_{2}$. Let also $L^{+}$ denote the positive limit set of $q(t)$ (see [6] for the definition of positive limit set). For any $p \in L^{+}$, there is a sequence $\left\{t_{n}\right\}$ with $t_{n} \rightarrow \infty$ such that $q\left(t_{n}\right) \rightarrow p$ as $n \rightarrow \infty$. It follows immediately from the continuity of $\mu_{1}$ and $\mu_{2}$, that $\mu_{1}(p)=a_{1}$ and $\mu_{2}(p)=a_{2}$.

It is desired now to show that $a_{1}=0$. If $a_{1}>0$, the invariant property of $L^{+}$(see Lemma 4.1 in [6]) along with the fact that $\mu_{1}(p)=a_{1}$ for any $p \in L^{+}$and the nestedness property of the convex hull of the agents, results that starting from any $p(0)=\left(p_{1}(0), \ldots, p_{n}(0)\right) \in L^{+}$, the convex hull $S(t)$ will remain fixed, i.e. $S(t) \equiv S(0)$. Consider an agent, say agent $i$, at a vertex of $S(0)$, and let $l_{1}$ and $l_{2}$ be the two lines obtained by extending the two edges connected to this vertex on the boundary of $S(0)$. Now, it results from Lemma 4 (once with $l=l_{1}$ and then with $\left.l=l_{2}\right)$ that either agent $i$ moves away from this vertex, or $f_{l_{1}}\left(\dot{p}_{i}\right) \equiv f_{l_{2}}\left(\dot{p}_{i}\right) \equiv 0$; the latter case implies that agent $i$ stays fixed at that vertex. Thus, in order for $S(t)$ to remain fixed, there should be at least one fixed agent at each vertex of $S(0)$, which contradicts Assumption 2. This contradiction yields $a_{1}=0$, i.e., if $p=\left(p_{1}, \ldots, p_{n}\right) \in L^{+}$, then $p_{i}$ 's are collinear. Using this property and an argument similar to the one given above, it is concluded that $a_{2}=0$. To complete the proof, note that since $S(t)$ is nested, it satisfies the finite intersection property, and hence according to Theorem 1 in [5], page 136, $\bigcap_{t \geq 0} S(t)=Q \neq \emptyset$. On the other hand, $a_{2}=0$ implies that the diameter of $S(t)$ approaches 0 as $t \rightarrow \infty$, which means that $Q$ is a single point. Furthermore, $Q \in S(t)$ yields $\left\|q_{i}(t)-Q\right\| \leq \mu_{2}(q(t))$ and this, in turn, implies that $q_{i}(t) \rightarrow Q$ as $t \rightarrow \infty$ because $\mu_{2}(q(t)) \rightarrow 0$ as $t \rightarrow \infty$. This completes the proof of the convergence of the agents to a fixed single point.

Assumption 2 is essential in the above theorem, but it is not straightforward to verify it, in general. The following proposition will prove useful in verifying the condition of this assumption.

Proposition 1 Let the condition of Assumption 1 hold, and assume the convex hull of the agents is fixed. Then for a fixed agent, say agent $i$, at a vertex of this convex hull, and for every $j \in N_{i}$, either $q_{j} \equiv q_{i}$ or $\beta_{i j} \equiv 0$.

Proof. This can be proved by using Corollary 1 for the two lines passing through the two edges on the boundary of the convex hull connected to the vertex at which $q_{i}$ is fixed, and is omitted due to space limitations.

The main advantage of this work over $[7,8]$ is described in the next proposition.

Proposition 2 Consider a set of $n$ agents in the 2D plane with the dynamics of the form (1), and with a quasi-strongly connected information flow graph. Let the control law be of the form (2), where the corresponding coefficients are assumed to meet the conditions of Assumption 1. Define $Q_{i}=\left\{q_{j} \mid j \in N_{i} \cup\{i\}\right\}$, and assume that if agent $i$ is at a vertex of $\operatorname{Conv}\left(Q_{i}\right)$ and $Q_{i}$ is not a singleton, then $\dot{q}_{i} \not \equiv 0$. Then the agents converge to a consensus.

Proof. It suffices to show that the conditions of the proposition imply that Assumption 2 holds. Suppose that there is a solution for which Assumption 2 does not hold, and let agent $i$ be a fixed agent at a vertex of the convex hull for such a solution. Clearly, $q_{i}$ is also a vertex of $\operatorname{Conv}\left(Q_{i}\right)$ at all times. This, along with the fact that $\dot{q}_{i} \equiv 0$, implies that $Q_{i}$ should be a singleton at all times, and hence $q_{j} \equiv q_{i}$ for all $j \in N_{i}$. Repeating the same argument, one can conclude that $q_{j} \equiv q_{i}$ for any agent $j$ from which $i$ is reachable in $G$. Now, consider two fixed agents $i_{1}$ and $i_{2}$ at two distinct vertices of the convex hull. Since $G$ is quasi-strongly connected, there exists an agent from which both $i_{1}$ and $i_{2}$ are reachable in $G$, implying that $q_{i_{1}} \equiv q_{i_{2}}$. This contradicts the assumption that agents $i_{1}$ and $i_{2}$ are located at two distinct vertices of the convex hull, and hence completes the proof.

Remark 1 [7,8] do not guarantee the convergence to a consensus under the setting of Proposition 2. More precisely, $[7,8]$ require $\dot{q}_{i} \neq 0$ instead of $\dot{q}_{i} \not \equiv 0$ (in the statement of the 
proposition) to deduce the convergence to a consensus while the above proposition allows agent $i$ at a vertex of $\operatorname{Conv}\left(Q_{i}\right)$ to attain zero velocity (even if $Q_{i}$ is not a singleton) as long as it is not fixed. The only limitation here, however, is that $\beta_{i j}$ 's need to be analytic, while there is not such a constraint in $[7,8]$ (it is only required there that the $u_{i}$ 's are continuous functions of the state).

\section{Simulation Results}

Consider $n$ agents with the dynamics of the form (1) moving in a $2 \mathrm{D}$ plane with local control laws given by

$$
\begin{aligned}
u_{i}= & -\left(\left\|q_{i}-q_{1}\right\|^{2}-c_{i}^{2}\right)^{2}\left(q_{i}-q_{1}\right) \\
& -\left(\left\|q_{i}-q_{i+1}\right\|^{2}-c_{1}{ }^{2}\right)^{2}\left(q_{i}-q_{i+1}\right), \quad 2 \leq i \leq n
\end{aligned}
$$

where $q_{n+1}=q_{2}$, and $c_{i}$ 's, $i \in \mathbb{N}_{n}$, are distinct nonnegative constants satisfying $0 \leq c_{i}<\frac{c_{1}}{2}$, for $i=2, \ldots, n$. Assume also that agent 1 is a static leader, i.e. $u_{1} \equiv 0$. Assumption 1 is clearly satisfied for the coefficients corresponding to the given control law. Suppose that there exists a solution with the given control law for which Assumption 2 does not hold. Denote with $\mathscr{I}$ the set of fixed agents at the vertices of the convex hull for this solution. Proposition 1 implies that for any $i \in \mathscr{I}$, if $q_{i} \not \equiv q_{1}$ then $\left\|q_{i}-q_{1}\right\| \equiv c_{i}$. Let $d$ denote the diameter of the convex hull. Then, $d=\max _{r, s \in \mathscr{I}}\left\{\| q_{r}-\right.$ $\left.q_{s} \|\right\} \leq \max _{r, s \in \mathscr{I}}\left\{\left\|q_{r}-q_{1}\right\|+\left\|q_{s}-q_{1}\right\|\right\}<\frac{c_{1}}{2}+\frac{c_{1}}{2}=c_{1}$. Now, consider an agent $i \in \mathscr{I}$ for which $q_{i} \not \equiv q_{1}$. The relation $\left\|q_{i}-q_{i+1}\right\| \leq d<c_{1}$ along with Proposition 1 yields $q_{i+1} \equiv q_{i}$. This means that $q_{i+1}$ is also fixed and $q_{i+1} \not \equiv q_{1}$; hence, as shown earlier $\left\|q_{i+1}-q_{1}\right\| \equiv c_{i+1}$. This is a contradiction since $\left\|q_{i+1}-q_{1}\right\| \equiv\left\|q_{i}-q_{1}\right\| \equiv c_{i}$ and $c_{i} \neq c_{i+1}$. Therefore, both Assumptions 1-2 hold and the convergence to a consensus is deduced from Theorem 2. It is easy to verify that the convergence to a consensus for this example cannot be deduced from $[7,8]$. The trajectories of the agents for the case of $n=5$ are depicted in Fig. 2. The corresponding values of $c_{i}$ 's, $i \in \mathbb{N}_{5}$, are chosen to be $1, \frac{1}{3}, \frac{1}{4}, \frac{2}{5}$, and $\frac{1}{5}$, respectively. The convex hull of the agents at three time instants $t_{0}=0 \mathrm{sec}, t_{1}=0.03 \mathrm{sec}$, and $t_{2}=0.43 \mathrm{sec}$ are also drawn in Fig. 2, which confirm the nestedness property of $S(t)$ shown in Theorem 1.

\section{Conclusions}

This paper deals with a class of continuous-time nonlinear consensus algorithms for single integrator agents. It is assumed that the information flow graph is static and directed. It is also assumed that the control input of each agent is a state-dependent combination of the relative positions of its neighbors in the information flow graph. Sufficient conditions are then derived for the convergence of the agents to a common point. Under these conditions, it is shown that the convex hull of the agents has a contracting property. The convergence is subsequently proved using a LaSalle-like approach as well as the finite intersection property of the convex hull. The criteria obtained here are shown to be more

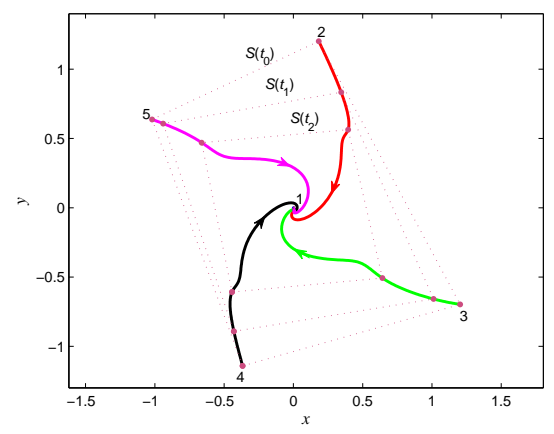

Fig. 2. The agents' planar motion for the case of $n=5$.

general than the existing results in the literature. This is also illustrated by a leader-based consensus example.

\section{References}

[1] Amir Ajorlou, Ahmadreza Momeni, and Amir G. Aghdam. A class of bounded distributed control strategies for connectivity preservation in multi-agent systems. Accepted for publication in IEEE Trans. on Autom. Contr., 2010.

[2] Dimos V. Dimarogonas and Kostas J. Kyriakopoulos. Connectedness preserving distributed swarm aggregation for multiple kinematic robots. IEEE Trans. on Robotics, 24(5):1213-1223, 2008.

[3] Michel Gondran and Michel Minoux. Graphs and Algorithms. John Wiley \& Sons Inc., New York, 1984.

[4] Meng Ji and Magnus Egerstedt. Distributed coordination control of multiagent systems while preserving connectedness. IEEE Trans. on Robotics, 23(4):693-703, 2007.

[5] John L. Kelley. General Topology. Springer, New York, 1975.

[6] Hassan K. Khalil. Nonlinear Systems. Prentice Hall, New Jersey, 3 edition, 2002.

[7] Zhiyun Lin, Bruce A. Francis, and Manfredi Maggiore. On the state agreement problem for multiple nonlinear dynamical systems. In Proc. of 16th IFAC World Congress, 2005.

[8] Zhiyun Lin, Bruce A. Francis, and Manfredi Maggiore. State agreement for continuous-time coupled nonlinear systems. SIAM Journal on Control and Optimization, 46(1):288-307, 2007.

[9] Luc Moreau. Stability of continuous-time distributed consensus algorithms. In Proc. of the 43th IEEE Conf. on Decision and Control, pages 3998-4003, 2004.

[10] Luc Moreau. Stability of multiagent systems with time-dependent communication links. IEEE Trans. on Autom. Contr., 50(2):169-182, 2005.

[11] Reza Olfati-Saber. Flocking for multi-agent dynamic systems: Algorithms and theory. IEEE Trans. on Autom. Contr., 51(3):401420, 2006

[12] Reza Olfati-Saber, J. Alex Fax, and Richard M. Murray. Consensus and cooperation in networked multi-agent systems. Proc. of the IEEE, 95(1):215-233, 2007.

[13] Reza Olfati-Saber and Richard M. Murray. Consensus problems in networks of agents with switching topology and time-delays. IEEE Trans. on Autom. Contr., 49(9):1520-1533, 2004.

[14] Wei Ren and Randal W. Beard. Consensus seeking in multiagent systems under dynamically changing interaction topologies. IEEE Trans. on Autom. Contr., 50(5):655-661, 2005.

[15] Herbert G. Tanner, Ali Jadbabaie, and George J. Pappas. Flocking in fixed and switching networks. IEEE Trans. on Autom. Contr., 52(5):863-868, 2007. 